\title{
Exome sequencing reveals SCO2 mutations in a family presented with fatal infantile hyperthermia
}

\author{
Nyamkhishig Sambuughin ${ }^{1}$, Xinyue Liu $^{2}$, Sunita Bijarnia ${ }^{3}$, Tarina Wallace ${ }^{1}$, Ishwar C Verma ${ }^{3}$, \\ Susan Hamilton ${ }^{4}$, Sheila Muldoon ${ }^{1}$, Luke J Tallon ${ }^{2}$ and Shuishu Wang ${ }^{5}$ \\ We applied whole-exome sequencing (WES) for identification of an underlying genetic cause of a disease in a family presented \\ with fatal infantile hyperthermia. Analysis of WES results revealed novel, deleterious compound missense mutations, Val160Ala \\ and Pro233Thr, in the synthesis of cytochrome C oxidase 2 gene (SCO2) encoding a mitochondrial protein, Sco2, which is \\ important for cytochrome C oxidase (COX) synthesis. Autosomal recessive mutations in SCO2 are known to be associated with \\ COX deficiency recognized as fatal infantile cardio-encephalomyopathy (604272, OMIM). The Val160Ala and Pro233Thr \\ mutations occurred in the conserved thioredoxin domain of $\mathrm{ScO} 2$ and predicted to disrupt protein folding and interaction \\ of Sco2 with other proteins. Our results show applicability of WES in identification of disease-causing mutations and in \\ establishing molecular diagnosis of severe, infantile onset disorder with a challenging diagnosis. \\ Journal of Human Genetics (2013) 58, 226-228; doi:10.1038/jhg.2012.156; published online 31 January 2013
}

Keywords: exome sequencing; fatal infantile cardio-encephalomyopathy; malignant hyperthermia; recessive mutation; synthesis of cytochrome $\mathrm{C}$ oxidase 2

\section{INTRODUCTION}

Whole-exome sequencing (WES) has proven to be an extremely valuable and cost-effective tool for uncovering disease genes and establishing molecular diagnosis in many rare disorders. ${ }^{1,2}$ This technology is particularly important for atypical, sporadic cases and small families for which the underlying genetic cause is not identified by traditional genetic studies.

In this study, we report a successful application of WES for identification of an underlying genetic cause of a disease in a family with a history of fatal infantile hyperthermia. We highlight the utility of WES in determining a molecular diagnosis when a detailed phenotype and clinical diagnosis of the patient was limited. The molecular diagnosis was based on the finding of novel, likely deleterious compound missense mutations, Val160Ala and Pro233Thr, in the synthesis of cytochrome $\mathrm{C}$ oxidase 2 gene $(\mathrm{SCO} 2){ }^{3}{ }^{3} \mathrm{SCO} 2$ encodes a metallochaperone, Sco2, which has an essential role in the biogenesis of cytochrome $\mathrm{C}$ oxidase $(\mathrm{COX}){ }^{4}$ Autosomal recessive mutations in $\mathrm{SCO} 2$ associated with $\mathrm{COX}$ deficiency known as fatal infantile cardio-encephalomyopathy $(604272$, OMIM $) .^{3-7}$

\section{MATERIALS AND METHODS}

An Indian couple who have lost three children was referred for a genetic study of malignant hyperthermia $(\mathrm{MH})$ because of the children's suspected history of persistent hyperthermia in a hot environment. The marriage was nonconsanguineous, pregnancy was normal and neonatal complications were denied (Supplementary Table S1). All children were male, deceased at 5, 9 and 10 months of age. The hyperthermia started about 1-2 months before death, and was continuous $\left(>39^{\circ} \mathrm{C}\right)$ and not associated with any major infection. None of the children were investigated for autopsy or muscle biopsy. The grandparents and parents were consented to participate in the genetic study (Figure 1a). The DNA from the last deceased boy was available. This study was approved by the Institutional Review Board of Uniformed Services University.

A family trio, consisting of parents and a deceased proband, was subjected to WES. Details of WES and data analysis are given in the Supplementary Information. Complete sequencing of the ryanodine receptor type 1 gene (RYR1) was performed in the deceased proband as described. ${ }^{8}$

\section{RESULTS}

Dominant mutations in RYR1 are associated with $\mathrm{MH}$, a pharmacogenetic disease of muscle, in which hyperthermia, among a constellation of other symptoms and signs, occurs on exposure to anesthetic agents. ${ }^{9,10}$ Although $\mathrm{MH}$ is a drug-induced disorder, cases of sudden death during heat challenge, and febrile illness in the absence of triggering anesthetics have been reported. ${ }^{8,11}$ Therefore, we analyzed entire RYR1 in the deceased proband and the results were negative. Although the degree of hyperthermia was impressive in the proband, stiffness and rigidity typical of $\mathrm{MH}$ crises were not observed (Supplementary Table S1). In addition, the proband responded to

${ }^{1}$ Department of Anesthesiology, Uniformed Services University, Bethesda, MD, USA; ${ }^{2}$ Institute for Genome Sciences, University of Maryland School of Medicine, Baltimore, MD, USA; ${ }^{3}$ Center of Medical Genetics, Sir Ganga Ram Hospital, New Delhi, India; ${ }^{4}$ Department of Molecular Physiology and Biophysics, Baylor College of Medicine, Houston, TX, USA and ${ }^{5}$ Department of Biochemistry and Molecular Biology, Uniformed Services University, Bethesda, MD, USA

Correspondence: Dr N Sambuughin, Department of Anesthesiology, Uniformed Services University, 4301 Jones Bridge Road, Bethesda 20814, MD, USA E-mail: nyamkhishig.sambuughin@usuhs.edu

Received 9 October 2012; revised 6 December 2012; accepted 19 December 2012; published online 31 January 2013 
a

b

Sequence: CTGCACTGG CAACCCTGA

Wild type:
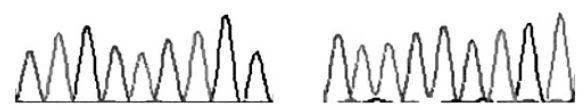

Mutant:
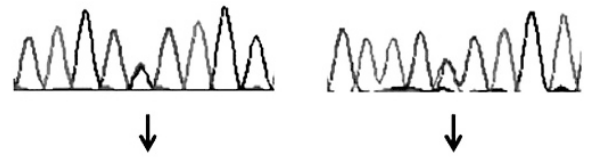

c

$\mathrm{SCO} 2$ :

V160A

*

P233T

$\begin{array}{lll}\text { Human: } & \text { PGLPPVQPVFIT } & \text { IYLLNPDGLFT } \\ \text { Dog: } & \text { PGLPPVQPVFIT } & \text { IYLLSPDGLFT } \\ \text { Mouse : } & \text { PDLPLVQPVFIT } & \text { IYLLNPDGLFT } \\ \text { Zebra fish: } & \text { PSLPSVQPLFIT } & \text { IYLVNPDGLFI }\end{array}$

Figure 1 (a) Pedigree of a family. Filled symbols with bisecting lines indicate individuals died under similar circumstances. An arrow denotes a proband that was compound heterozygous for Val160Ala and Pro233Thr mutations. Symbol with star indicates Val160Ala mutation carrier; symbols with dot indicate Pro233Thr mutation carriers; empty symbols indicate clinically healthy family members with no mutation. (b) Sequence chromatographs of $\mathrm{SCO} 2$ in control and case. Arrows show position of nucleotide changes those results in Val160Ala and Pro233Thr mutations. (c) Alignment of the region of Sco2 mutations and flanking residues across species. Sco2 mutations are shown on top of the alignment. The mutated residues are in red. A full color version of this figure is available at the Journal of Human Genetics journal online.

antipyretics and his blood gas results revealed respiratory alkalosis. These signs are not consistent with clinical findings in $\mathrm{MH}^{9}{ }^{9}$ As a result of the proband's metabolic acidosis, we hypothesized that the proband is likely to suffer from a rare metabolic disorder and performed WES on the proband and his parents. WES resulted in $>81 \%$ of the targets reaching $>30 \times$ coverage (Supplementary Table S2). We used analysis pipeline to select missense single-nucleotide polymorphisms and coding insertions-deletions that are not present in publicly available exome databases ${ }^{12,13}$ and determined variants impact with PolyPhen and SIFT. WES results for other causative genes for $\mathrm{MH}$ including CACNA1S, CASQ1 and CACNA2D1 were negative. We further identified six compound heterozygous and two homozygous variants under autosomal recessive inheritance (Supplementary Table S3). Of these, compound heterozygous variants in three genes, ANKD35, SCO2 and XAB2, were noteworthy as they introduced missense mutations with damaging effects. However, only both Val160Ala and Pro233Thr mutations in SCO2 were absent in the publicly available data on exome sequencing of $>7500$ European and African Americans. ${ }^{12,13}$ SCO2 encodes a mitochondrial protein, $\mathrm{Sco} 2$,

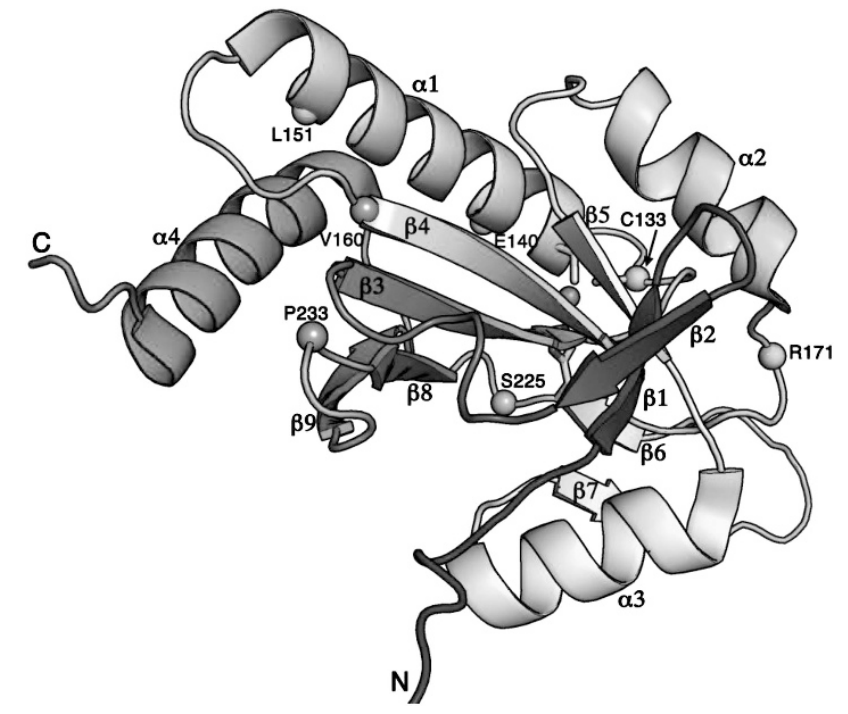

Figure 2 Pathogenic mutations in human $\mathrm{SCO} 2$ mapped on the protein structure. The ribbon diagram was prepared from PDB file 2RLI (Bianci et $a . .^{14}$ ), and colored in a rainbow spectrum with blue to red from $\mathrm{N}$ - to $\mathrm{C}$-terminus. Five known pathogenic mutation sites are labeled and are marked with spheres colored identically to the ribbon diagram. The two new mutations identified in this study are marked with gray spheres. The bound $\mathrm{Cu}(\mathrm{I})$ is shown as a red sphere, and its coordinating side chains are shown as sticks. The figure was prepared with PyMOL molecular graphics system (Schrödinger, LLC). A full color version of this figure is available at the Journal of Human Genetics journal online.

which participates in COX assembly by providing copper to the catalytic CuA site on COX subunit II. ${ }^{4}$ Recessive mutations in $\mathrm{SCO} 2$ cause COX deficiency known as fatal infantile cardio-encephalopathy characterized by progressive hypertrophic cardiomyopathy in the neonatal period with respiratory insufficiency, hypotonia and metabolic acidosis. ${ }^{3-7}$ Death occurs during the first year of life mainly due to cardiac or respiratory failure. Inheritance of newly identified mutations was confirmed by Sanger sequencing (Figure 1a and b). Segregation analysis in extended family shows Val160Ala occurred de novo in the father and the mother to be a heterozygous carrier of the Pro233Thr mutation (Figure 1a).

The Val160Ala and Pro233Thr mutations are located in conserved thioredoxin domain of the protein and mutate residues that are conserved across species (Figure 1c). The Val160Ala mutation lies at the beginning of $\beta 4$ whereas the Pro233Thr is located in the loop between the last two $\beta$ strands of the protein (Figure 2). The side chain of Val160 is part of the hydrophobic core at the interface between helix $\alpha 1$ and the central $\beta$-sheet. ${ }^{14}$ This hydrophobic core also includes Leu151, mutation of which into a proline is one of known pathogenic missense mutations in the human SCO2. ${ }^{15}$ The Leu151Pro mutation is thought to weaken the interactions between helix $\alpha 1$ and the central $\beta$-sheet, thereby destabilizing the protein fold and increasing its turnover rate. ${ }^{14}$ Similarly, mutation of Val160Ala is likely to have the same effect on the protein stability by disrupting the same hydrophobic core. Pro233 is at the position $i+1$ of a $\beta$-turn, and the Pro233Thr mutation is likely to change the local structure at the turn and affect its interactions with the nearby loop between strands $\beta 2$ and $\beta 3$ (Figure 2). It is possible that the surface around Pro233 is involved in the interactions of Sco2 with other proteins, and thus the variation because of the mutation may result in the loss of protein-protein interaction and the loss of the Sco2 function. 
Sequences around Val160 and Pro233 are highly conserved among Sco2 proteins (Figure 1c), suggesting that this part of the protein is likely to have an important role in the Sco2 function.

\section{DISCUSSION}

This study reports unexpected finding of novel compound heterozygous SCO2 mutations, Val160Ala and Pro233Thr, in a proband referred for a genetic study of $\mathrm{MH}$. The patient presented with a clinical picture, fatal hyperthermia and metabolic acidosis, which is similar to $\mathrm{MH}$. However, his overall clinical signs, such as lack of rigidity during hyperthermia, response to antipyretics and respiratory alkalosis clearly differentiate the patient's metabolic crisis from $\mathrm{MH}$. Although some patients with mitochondrial defects manifests $\mathrm{MH}$ like reactions, or show increased susceptibility to $\mathrm{MH}$ by contracture testing $^{16,17}$ the majority of patients with mitochondrial myopathies do not develop $\mathrm{MH}$ on receiving triggering anesthetics, suggesting a lack of association between these two conditions. ${ }^{18}$

The Val160Ala and Pro233Thr mutations in SCO2 are likely to be deleterious. The Val160Ala mutation occurred at the same position with previously reported Val160Gly mutation in a 4-month-old girl with severe case of Sco2 deficiency. ${ }^{19}$ Mapping of both mutations on the protein structure shows that Val160Ala is likely to disturb the protein folding, whereas Pro233Thr may affect Sco2 interaction with other proteins. The structural perturbations from these mutations will probably lead to loss of Sco2 function; that is, indispensable for COXII synthesis. In addition, both mutations were absent in $>7500$ publicly available exomes with a diverse ethnic background. ${ }^{12,13}$ Based on these findings, we suggest disease diagnosis as fatal infantile cardio-encephalopathy, which was not considered in the referral of the patient.

To date, at least 43 cases of Sco 2 deficiency has been reported and all except 1 of them were patients with European ancestry. ${ }^{3,5-7,19,20}$ All patients of European ancestry carry Glu140Lys mutation either in the homozygous or compound heterozygous state. The only case of Sco2 deficiency not associated with Glu140Lys mutation was a patient with homozygous Gly193Ser mutation and the patient was born to consanguineous parents of Indian ancestry. ${ }^{19}$ Finding of another Indian family with Sco2 deficiency not associated with common European Glu140Lys mutation demonstrates a regional difference in the distribution of SCO2 mutations.

Limitation of this study is a lack of functional analysis establishing pathogenic relevance of newly identified SCO2 mutations. Further studies to determine the pathogenic consequence of $\mathrm{SCO} 2$ mutations are warranted.

\section{CONFLICT OF INTEREST}

The authors declare no conflict of interest.

\section{ACKNOWLEDGEMENTS}

We are grateful to the family for their participation in this study. We thank Dr W Watson, Department of Neurology, Uniformed Services University for valuable comments. The authors thank Biomedical Instrumentation Center of Uniformed Services University for oligo synthesis and Sanger sequencing.

1 Bamshad, M. J., Ng, S. B., Bigham, A. W., Tabor, H. K., Emond, M. J., Nickerson, D. A. et al. Exome sequencing as a tool for Mendelian disease gene discovery. Nat. Rev. Genet. 12, 745-755 (2011).

2 Majewski, J., Schwartzentruber, J., Lalonde, E., Montpetit, A. \& Jabado, N. What exome sequencing can do for you? J. Med. Genet. 48, 580-589 (2011).

3 Papadopoulou, L. C., Sue, C. M., Davidson, M. M., Tanji, K., Nishino, I., Sadlock, E. J. et al. Fatal infantile cardioencephalomyopathy with COX deficiency and mutations in SCO2, a COX assembly gene. Nat. Genet. 23, 333-337 (1999).

4 Leary, S. C., Sasarman, F., Nishimura, T. \& Shoubridge, E. A. Human SCO2 is required for the synthesis of $\mathrm{CO}$ II and as a thiol-disulphide oxidoreductase for SCO1. Hum. Mol. Genet. 18, 2230-2240 (2010)

5 Verdijk, R. M., de Krijger, R., Schoonderwood, K., Tiranti, V., Smeets, H., Govaerts, L. C. P. et al. Phenotypic consequences of a novel SCO2 gene mutation. Am. J. Med. Genet. 146, 2822-2827 (2008).

6 Joost, K., Rodenburg, R., Piirsoo, A., van den Heuvel, B., Zordania, R. \& Ōunap, K. A novel mutation in the SCO2 gene in a neonate with early-onset cardioencephalomyopathy. Pediatr. Neurol. 42, 227-230 (2010).

7 Pronicki, M., Kowalski, P., Piekutowska-Abramczuk, D., Taybert, J., KarkucinskaWieskowska, A., Szymanska-Debinska, T. et al. A homozygous mutation in the SCO2 gene causes a spinal muscular atrophy like presentation with stridor and respiratory insufficiency. Eur. J. Pediatr. Neurol. 14, 253-260 (2010).

8 Groom, L., Muldoon, S., Tang, Z., Brandom, B. W., Bayarsaikhan, M., Bina, S. et al. Identical de novo mutation in the RYR1 gene associated with fatal, stress-induced malignant hyperthermia in two unrelated families. Anesthesiology 115, 938-945 (2011).

9 Rosenberg, H., Sambuughin, N. \& Dirksen, R. T. Malignant hyperthermia susceptibility. in GeneReviews at GeneTests: Medical Genetics Information Resource [database online]. Copyright, University of Washington, Seattle, 1997-2012. Available at http://www.genetests.org.

10 Robinson, R. L., Carpenter, D., Shaw, M., Halsall, J. \& Hopkins, P. Mutations in RYR1 in Malignant hyperthermia and central core disease. Hum. Mutat. 27, 977-989 (2006).

11 Tobin, J., Jason, D. R., Challa, V. R., Nelson, T. E. \& Sambuughin, N. Malignant hyperthermia and apparent heat stroke. JAMA 286, 168-169 (2001).

12 Consortium TGP. A map of human genome variation from population scale sequencing. Nature 467, 1061-1073 (2010).

13 NHLBI Exome Sequencing Project. Seattle, WA, URL: http://evs.gs.washington.edu/ EVS/. Accessed 02 July 2012.

14 Bianci, L., Bertini, I., Ciofi-Baffoni, S., Gerothanassis, I. P., Leontari, I., Martnelli, M et al. A structural characterization of human SCO2. Structure 15, 1132-1140 (2007)

15 Sacconi, S., Salviati, L., Sue, C. M., Shanske, S., Davidson, M. M., Bonilla, E. et al. Mutation screening in patients with isolated cytochrome $c$ oxidase deficiency. Pediatr. Res. 53, 224-230 (2003).

16 Figarella-Branger, D., Kozak-Ribbens, G., Rodet, L., Aubert, M., Borsarelli, J., Cozzone, P. J. et al. Pathological findings in 165 patients explored for malignant hyperthermia susceptibility. Neuromusc. Disord. 3, 553-556 (1993).

17 Finsterer, J., Michalek-Sauberer, A. \& Hoftberger, R. Malignant hyperthermia susceptibility in a patient with mitochondrial disorder. Metab. Brain Dis. 24, 501-506 (2009).

18 Ross, A. K. Muscular dystrophy versus mitochondrial myopathy: the dilemma of the undiagnosed hypotonic child. Pediatr. Anesth. 17, 1-6 (2007).

19 Knuff, M., Faber, J., Huth, R. G., Freisinger, P., Zepp, F. \& Kampmann, C. Identification of a novel compound heterozygote SCO2 mutation in cytochrome c oxidase deficient fatal infantile cardiomyopathy. Acta. Pediatrica. 96, 128-134 (2007).

20 Mobely, B. C., Enns, G. M., Wong, L. J. \& Vogel, H. A novel homozygous SCO2 mutation, p.G193S, causing fatal infantile cardioencephalomyopathy. Clin. Neuropathol. 28, 143-149 (2009).

Supplementary Information accompanies this paper on Journal of Human Genetics website (http://www.nature.com/jhg) 